\title{
Relation of anomalous $F$ region radar echoes in the high-latitude ionosphere to auroral precipitation
}

\author{
Hanna Dahlgren ${ }^{1,2}$, Nicola M. Schlatter ${ }^{1}$, Nickolay Ivchenko ${ }^{1}$, Lorenz Roth ${ }^{1}$, and Alexander Karlsson ${ }^{1}$ \\ ${ }^{1}$ School of Electrical Engineering, Royal Institute of Technology KTH, Stockholm, Sweden \\ ${ }^{2}$ School of Physics and Astronomy, University of Southampton, Southampton, UK \\ Correspondence to: Hanna Dahlgren (hannad@kth.se)
}

Received: 20 January 2017 - Accepted: 28 February 2017 - Published: 22 March 2017

\begin{abstract}
Non-thermal echoes in incoherent scatter radar observations are occasionally seen in the high-latitude ionosphere. Such anomalous echoes are a manifestation of plasma instabilities on spatial scales matching the radar wavelength. Here we investigate the occurrence of a class of spatially localized anomalous echoes with an enhanced zero Doppler frequency feature and their relation to auroral particle precipitation. The ionization profile of the $\mathrm{E}$ region is used to parametrize the precipitation, with $\mathrm{nmE}$ and $\mathrm{hmE}$ being the E region peak electron density and the altitude of the peak, respectively. We find the occurrence rate of the echoes to generally increase with $\mathrm{nmE}$ and decrease with $\mathrm{hmE}$, thereby indicating a correlation between the echoes and high-energy flux precipitation of particles with a high characteristic energy. The highest occurrence rate of $>20 \%$ is found for $\mathrm{hmE}=109 \mathrm{~km}$ and $\mathrm{nmE}=10^{11.9} \mathrm{~m}^{-3}$, averaged over the radar observation volume.
\end{abstract}

Keywords. Ionosphere (auroral ionosphere; particle precipitation; plasma waves and instabilities)

\section{Introduction}

Incoherent scatter radars use thermal fluctuations of electrostatic waves in the ionosphere plasma to derive plasma parameters. Radar echoes above the thermal level are a manifestation of electrostatic instabilities (Foster et al., 1988; Rietveld et al., 1991; Collis et al., 1991). Evidence for strong Langmuir turbulence (SLT; e.g. Robinson, 1997; Briand, 2015) has been found in anomalous radar echoes (Isham et al., 2012; Akbari et al., 2012) in the form of enhanced zero Doppler frequency features in the ion line spectra and enhanced backscatter at the plasma frequency.
Common to observations of SLT radar signatures is the altitude from which the anomalous echoes arise, typically 200 to $300 \mathrm{~km}$, and their narrow altitude extent of a few kilometres to tens of kilometres (Akbari et al., 2013). Ekeberg et al. (2012) and Schlatter et al. (2014) studied the occurrence of the anomalous echoes by identifying the ion line signature of the echoes in a large dataset recorded with the European Incoherent Scatter Scientific Association (EISCAT) Svalbard radar (ESR). They found that the anomalous signatures frequently occurred for magnetically disturbed conditions and with a peak occurrence at around 21 MLT. Schlatter et al. (2014) found the highest occurrence, as measured over the radar beam width, to be $0.6 \%$ at 21 MLT and for a local $\mathrm{K}$ index of 5. Very little data were available for times with a $\mathrm{K}$ index above 6, giving insignificant statistics. A correlation of the echoes with high-energy electron precipitation was indicated by the local time distribution and $\mathrm{E}$ region electron density profiles derived from the incoherent scatter measurements (Schlatter et al., 2014).

The occurrence rates reported by Schlatter et al. (2014) suggest that anomalous echoes resembling SLT signatures are frequently observed with the ESR in the disturbed evening sector. Simultaneous magnetometer measurements indicate magnetic disturbances and, thus, possible particle precipitation on a large spatial scale. However, the magnetometer measurements cannot be unambiguously related to the specific volume covered by the radar measurements. It has not been investigated how often the anomalous echoes appear in regions of particle precipitation. Furthermore, the question arises under which conditions the echoes are observed and whether a specific type of precipitation can be identified as the source of the instability leading to these radar echoes and possible SLT. 
Table 1. Investigated data intervals and events in $6 \mathrm{~s}$ resolution data.

\begin{tabular}{lrr}
\hline Start time UT & Length (h) & Events \\
\hline 1 Oct 2007 00:00 & 24 & 121 \\
21 Jan 2008 00:00 & 24 & 68 \\
7 Mar 2007 15:00 & 3 & 55 \\
16 Mar 2007 21:00 & 3 & 34 \\
2 Apr 2007 21:00 & 3 & 0 \\
10 Apr 2007 15:00 & 3 & 0 \\
29 Apr 2007 18:00 & 3 & 0 \\
27 May 2007 15:00 & 3 & 0 \\
4 Jul 2007 19:00 & 3 & 0 \\
22 Sep 2007 18:00 & 3 & 34 \\
29 Sep 2007 19:00 & 3 & 35 \\
30 Sep 2007 18:00 & 3 & 43 \\
4 Oct 2007 17:00 & 3 & 33 \\
5 Jan 2008 19:00 & 3 & 3 \\
\hline
\end{tabular}

Here we investigate the relation of the SLT-like anomalous radar echoes to auroral precipitation. We study the $\mathrm{E}$ region peak density and peak altitude for a large number of events where anomalous radar echoes were detected to gain insight into prevailing conditions and the origin of the echoes.

\section{Data}

During the International Polar Year (IPY; 2007-2008), the ESR conducted nearly continuous observations. The radar was observing in the direction of magnetic zenith, and ion line data were taken with a range resolution of $4.75 \mathrm{~km}$ and a radar integration period, further referred to as a dump, of $6 \mathrm{~s}$ (for more details see, e.g., Schlatter et al., 2014). Data from the nearby located Longyearbyen magnetometer (LYR) are used to evaluate geomagnetic conditions.

A manual selection of a data subset was conducted from the complete IPY dataset for this detailed study. Two complete days (1 October 2007 and 21 January 2008) with a high occurrence of the anomalous echoes are analysed. In addition, twelve $3 \mathrm{~h}$ long intervals were selected in the afternoon magnetic local time sector from other days when the local $\mathrm{K}$ index was above 5 . These data intervals were expected to contain the anomalous echoes based on the statistical findings of the automated search of Schlatter et al. (2014), and were still limited enough, rendering themselves useful for the careful manual analysis as described below. A list of the investigated data intervals is given in Table 1.

\section{Analysis and results}

A manual search was conducted for anomalous echoes with enhanced backscatter power at the zero Doppler shift (following the characteristics of the class of events reported by

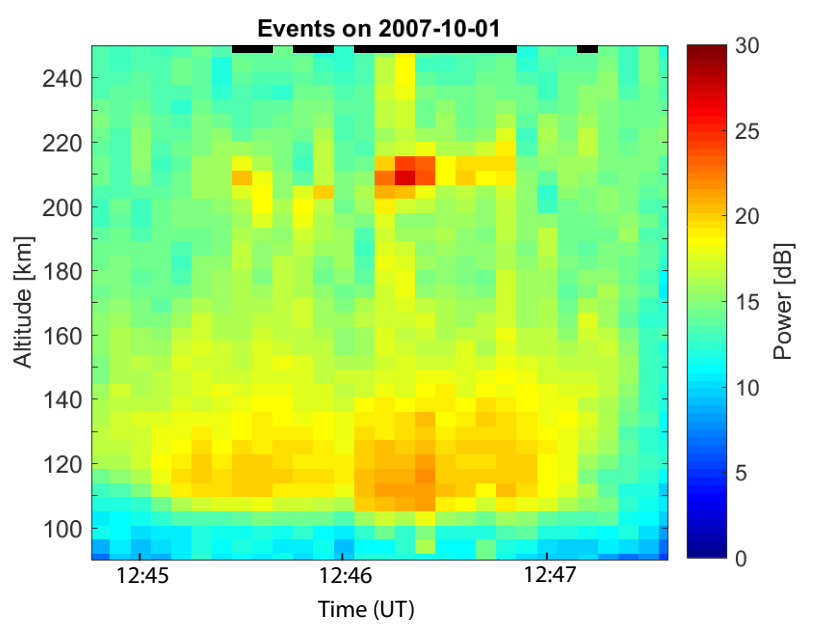

Figure 1. Received backscattered power as a function of time and altitude on 1 October 2007. In addition to the $\mathrm{E}$ region ionization, strong backscatter enhancements are found in a thin layer at $\sim 210 \mathrm{~km}$ altitude. The data dumps with detected anomalous echoes are marked with black bars at the top of the figure.

Isham et al., 2012 and Schlatter et al., 2014). The events were identified in a two-step selection. The first step is based on detecting radar return power enhancements that are narrow in range. Figure 1 shows an example from a period of $3 \mathrm{~min}$ on 1 October 2007. The variation in backscattered power at E region altitudes (between 100 and $150 \mathrm{~km}$ ) is the result of ionization by particle precipitation. At an altitude of $210 \mathrm{~km}$ intermittent enhancements in backscattered power are observed from a thin altitude layer indicating non-thermal radar backscatter. Enhancements of at least $20 \%$ over the neighbouring gates were selected (the selected time intervals in the 3 min interval shown in Fig. 1 are marked with black bars at the top of the figure) and analysed in the second step based on the shape of the ion line spectra. To classify as an event for this study, the spectrum for a given range-time interval was required to have an enhancement of both shoulders of the ion lines as well as a distinct peak with a zero Doppler shift. An example of such an ion spectrum can be seen in Fig. 3 in Schlatter et al. (2014). The measured increase in power of the echoes ranges from the threshold of 1.2 up to 30 times the background level. The enhancements may be even larger, as the echoes are likely more localized than the spatial and temporal resolution of the IPY experiment.

Anomalous echoes were identified in 426 dumps, each corresponding to a $6 \mathrm{~s}$ period, in the following referred to as events. Table 1 gives a list of the number of events in each investigated data period. For comparison, 87 events were identified in the same data by automatic identification (Schlatter et al., 2014), which used much more conservative criteria.

Electron density profiles obtained during the events indicate energetic electron precipitation, manifested by $\mathrm{E}$ region ionization. While the derivation of the $\mathrm{F}$ region parameters 


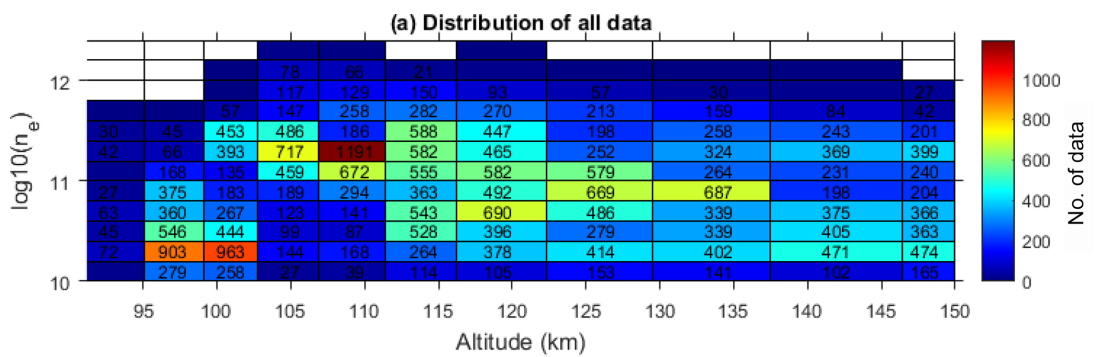

(b) Distribution of events

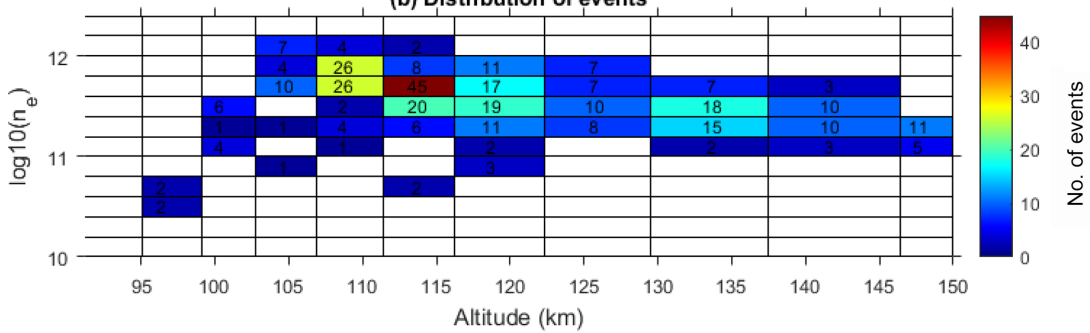

(c) Normalized

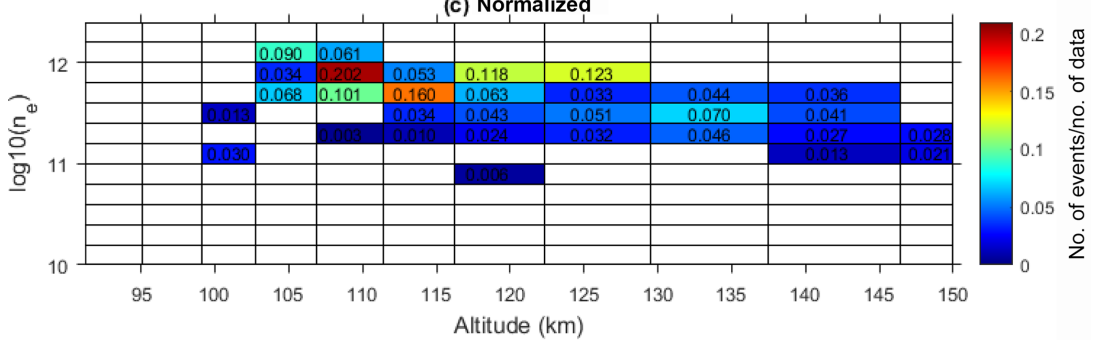

Figure 2. Distribution of E region peak altitude and peak electron density for (a) all investigated data and (b) events. In (c) the event distribution normalized by the data distribution is shown. The counts are also given in each bin.

from the radar data is affected by the anomalous echoes, the $\mathrm{E}$ region parameters are not, and so reliable density profiles can still be obtained in the $\mathrm{E}$ region. The height of the $\mathrm{E}$ region electron density peak, $\mathrm{hmE}$, is a measure of the characteristic energy of particle precipitation, and the peak electron density, nmE, is related to the energy flux. The event distribution as a function of $\mathrm{hmE}$ and $\mathrm{nmE}$ therefore gives insight into the characteristics of the electron precipitation during the anomalous radar echoes.

To retrieve the electron density altitude profiles from the radar observations, radar data were integrated over three dumps, $18 \mathrm{~s}$, and standard analysis was applied (Lehtinen and Huuskonen, 1996). The E region electron peak density and peak altitude were then extracted from these profiles. The observations were binned by $\mathrm{hmE}$ and $\mathrm{nmE}$ to investigate the occurrence of the anomalous echoes for different conditions.

Figure 2a shows the distribution of all data points binned by $\mathrm{hmE}$ and $\mathrm{nmE}$, with the number of dumps shown inside each of the bins. Figure $2 b$ shows the distribution of the identified events over the same bins. The majority of the events occur for $\mathrm{hmE}=100-145 \mathrm{~km}$ and $\mathrm{nmE}=10^{11}-10^{12} \mathrm{~m}^{-3}$. Figure $2 \mathrm{c}$ shows the event distribution normalized by the data coverage. Bins with fewer than three events are not shown to avoid the points with poor statistics. After the normaliza- tion, the bins with a high anomalous echo occurrence rate are concentrated in the parameter space region with high peak electron densities and low altitudes of the peak, characteristic of high-energy, high-flux precipitation. The peak occurrence rate is for the bin centred at $\mathrm{nmE}=10^{11.9} \mathrm{~m}^{-3}$ and $\mathrm{hmE}=109.1 \mathrm{~km}$ with anomalous echoes in $20 \%$ of the measurements.

\section{Discussion and conclusions}

We have found that the SLT-like anomalous radar echoes from the F region around $200-300 \mathrm{~km}$ altitude frequently occur during high-energy flux precipitation of particles with a high characteristic energy. The $\mathrm{hmE}$ and $\mathrm{nmE}$ values for which the occurrence maximizes are characteristic for electron precipitation with an energy flux of the order of $10 \mathrm{~mW} \mathrm{~m}^{-2}$ and a peak energy of about $3-10 \mathrm{keV}$ (e.g. Rees, 1989; Dahlgren et al., 2011). However, the exact relation between $\mathrm{nmE}$ and $\mathrm{hmE}$ and flux and energy depends on the profile of the atmospheric density and composition (atomic to molecular ratio), which varies with season, time of day and geomagnetic activity. Moreover, in the auroral region empirical models exhibit large deviation for individual events (e.g. Shepherd et al., 1995; Rishbeth et al., 2004). 
This study is complementary to that of Schlatter et al. (2014). While they applied an automated routine on a very large dataset to obtain the large-scale occurrence of the events as a function of magnetic local time and activity level, here we study the relation of the mechanism behind the plasma instability to auroral precipitation, detecting a strong connection between the two. In the large dataset study, it is important to keep the number of false detections down, hence the strategy of applying a high threshold and manually validating the automatically detected events. This gives reliable, but conservative results. In this study of the subset of the data, we can ensure positive detection of all the events, including somewhat weaker ones, which nonetheless exhibit the same basic characteristics (localized in altitude ion line enhancement with a central feature). This indicates that the plasma instability leading to this class of enhancements is rather common during energetic auroral precipitation.

The anomalous radar echoes discussed in this study are different from classical naturally enhanced ion acoustic lines (NEIALs) previously discussed by, e.g., Foster et al. (1988), Rietveld et al. (1991), and Grydeland et al. (2004). NEIALs are observed as enhancement of ion line shoulders over a wide altitude range (several hundred kilometres), with several order-of-magnitude increases of one or both ion line shoulders - and no features with a zero Doppler shift. While NEIALs have been found to be related to soft precipitation, both statistically (Ogawa et al., 2011) and on an event basis (e.g. Sullivan et al., 2008), the SLT-like anomalous echoes in this study were found to be closely related to energetic precipitation, characteristic of discrete aurora. This is in accordance with the event study of a similar zero Doppler shift feature seen during high-energy precipitation discussed by Schlatter et al. (2013). However, modelling studies (Isham et al., 2012; Akbari et al., 2016) point to the low energy part of the electron spectrum as being efficient in exciting the Langmuir turbulence. The electrostatic instability may be coupled to energy-degraded and secondary electron populations, rather than the primary precipitation. This may be due to either observational selectivity or physical mechanisms at play.

There are several observational constraints imposed by the data used. First of all, the radar observes only one value of the wave vector spectrum of the plasma density disturbance. The instability may cover a larger volume in space and/or a larger extent in the wave vector space than is readily observable by the radar. Second, the radar observations average over the illuminated volume and the integration time, thus underestimating the scattering enhancement if it is localized or intermittent. High-resolution observations with synthetic aperture interferometry would be needed to resolve this (see, e.g., Grydeland et al., 2005; Schlatter et al., 2015), but to our knowledge such observations have not yet been reported for the type of enhancement discussed here. Finally, the geometric effect of the radar beam covering a larger area in the $\mathrm{F}$ region compared to the $\mathrm{E}$ region means that the appearance of the location of the precipitation (guided by the magnetic field, with much smaller divergence between the $\mathrm{E}$ and the $F$ regions) in the two regions may differ: the regions at the edge of the radar beam in the $\mathrm{F}$ region map outside the beam in the $\mathrm{E}$ region and may feature different precipitation from that derived from the observed $\mathrm{E}$ region profile. This effect will smear out the statistical dependence in cases when auroral precipitation is structured in space. In particular it may explain the cases when events appear on the edges of auroral precipitation (as reported in, e.g., Schlatter et al., 2014).

It is not yet resolved what drives the SLT in the ionosphere, but the observations discussed here indicate a correlation between SLT and electron precipitation with an energy of at least a few kilo-electronvolts.

Data availability. Radar data for the IPY 2007-2008 are available through the EISCAT data portal, at https://www.eiscat.se/schedule/ schedule.cgi. The magnetometer data can be obtained through the Troms $\varnothing$ Geophysical Observatory data portal, at http://flux.phys.uit. no/geomag.html.

Competing interests. The authors declare that they have no conflict of interest.

Acknowledgements. Hanna Dahlgren was supported by the Swedish Research Council under grant 350-2012-6591. Lorenz Roth was supported by VINNOVA grant 2014-01459 and the Göran Gustafsson foundation. EISCAT is an international association supported by research organizations in China (CRIRP), Finland (SA), Japan (NIPR and STEL), Norway (NFR), Sweden (VR), and the United Kingdom (NERC). Operation of the EISCAT Svalbard radar during the IPY was supported by the Norwegian Research Council (NFR), through the IPY-ICESTAR project $176045 /$ S30, and the USA (NSF). Data from the Longyearbyen magnetometer were provided by the Troms $\varnothing$ Geophysical Observatory, Norway.

The topical editor, S. Milan, thanks one anonymous referee for help in evaluating this paper.

\section{References}

Akbari, H., Semeter, J. L., Dahlgren, H., Diaz, M., Zettergren, M., Strømme, A., Nicolls, M. J., and Heinselman, C.: Anomalous ISR echoes preceding auroral breakup: Evidence for strong Langmuir turbulence, Geophys. Res. Lett., 39, L03102, doi:10.1029/2011GL050288, 2012.

Akbari, H., Semeter, J. L., Nicolls, M. J., Broughton, M., and LaBelle, J. W.: Localization of auroral Langmuir turbulence in thin layers, J. Geophys. Res.-Space, 118, 3576-3583, doi:10.1002/jgra.50314, 2013.

Akbari, H., Guio, P., Hirsch, M. A., and Semeter, J. L.: Zakharov simulations of beam-induced turbulence in the au- 
roral ionosphere, J. Geophys. Res.-Space, 121, 4811-4825, doi:10.1002/2016JA022605, 2016.

Briand, C.: Langmuir waves across the heliosphere, J. Plasma Phys., 81, 325810204, doi:10.1017/S0022377815000112, 2015.

Collis, P. N., Häggström, L., Kaila, K., and Rietveld, M. T.: Eiscat radar observations of enhanced incoherent scatter spectra; Their relation to red aurora and field-aligned currents, Geophys. Res. Lett., 18, 1031-1034, doi:10.1029/91GL00848, 1991.

Dahlgren, H., Gustavsson, B., Lanchester, B. S., Ivchenko, N., Brändström, U., Whiter, D. K., Sergienko, T., Sandahl, I., and Marklund, G.: Energy and flux variations across thin auroral arcs, Ann. Geophys., 29, 1699-1712, doi:10.5194/angeo-291699-2011, 2011.

Ekeberg, J., Wannberg, G., Eliasson, L., and Häggström, I.: Solitoninduced spectrally uniform ion line power enhancements at the ionospheric F region peak, Earth Planets Space, 64, 605-611, doi:10.5047/eps.2012.02.005, 2012.

Foster, J. C., del Pozo, C., Groves, K., and St.-Maurice, J.-P.: Radar observations of the onset of current driven instabilities in the topside ionosphere, Geophys. Res. Lett., 15, 160-163, doi:10.1029/GL015i002p00160, 1988.

Grydeland, T., Blixt, E. M., Løvhaug, U. P., Hagfors, T., La Hoz, C., and Trondsen, T. S.: Interferometric radar observations of filamented structures due to plasma instabilities and their relation to dynamic auroral rays, Ann. Geophys., 22, 1115-1132, doi:10.5194/angeo-22-1115-2004, 2004.

Grydeland, T., Chau, J. L., La Hoz, C., and Brekke, A.: An imaging interferometry capability for the EISCAT Svalbard Radar, Ann. Geophys., 23, 221-230, doi:10.5194/angeo-23-221-2005, 2005.

Isham, B., Rietveld, M. T., Guio, P., Forme, F. R. E., Grydeland, T., and Mjølhus, E.: Cavitating Langmuir Turbulence in the Terrestrial Aurora, Phys. Rev. Lett., 108, 105003, doi:10.1103/PhysRevLett.108.105003, 2012.

Lehtinen, M. S. and Huuskonen, A.: General incoherent scatter analysis and GUISDAP, J. Atmos. Terr. Phys., 58, 435-452, doi:10.1016/0021-9169(95)00047-X, 1996.

Ogawa, Y., Buchert, S. C., Häggström, I., Rietveld, M. T., Fujii, R., Nozawa, S., and Miyaoka, H.: On the statistical relation between ion upflow and naturally enhanced ion-acoustic lines observed with the EISCAT Svalbard radar, J. Geophys. Res., 116, A03313, doi:10.1029/2010JA015827, 2011.
Rees, M. H.: Physics and Chemistry of the Upper Atmosphere, Cambridge University Press, Cambridge, UK, 1989.

Rietveld, M. T., Collis, P. N., and St.-Maurice, J.-P.: Naturally enhanced ion acoustic waves in the auroral ionosphere observed with the EISCAT 933-MHz radar, J. Geophys. Res., 96, 1929119305, doi:10.1029/91JA01188, 1991.

Rishbeth, H., Heelis, R. A., and Müller-Wodarg, I. C. F.: Variations of thermospheric composition according to AE-C data and CTIP modelling, Ann. Geophys., 22, 441-452, doi:10.5194/angeo-22441-2004, 2004.

Robinson, P. A.: Nonlinear wave collapse and strong turbulence, Rev. Mod. Phys., 69, 507-574, doi:10.1103/RevModPhys.69.507, 1997.

Schlatter, N. M., Ivchenko, N., Sergienko, T., Gustavsson, B., and Brändström, B. U. E.: Enhanced EISCAT UHF backscatter during high-energy auroral electron precipitation, Ann. Geophys., 31, 1681-1687, doi:10.5194/angeo-31-1681-2013, 2013.

Schlatter, N. M., Ivchenko, N., and Häggström, I.: On the relation of Langmuir turbulence radar signatures to auroral conditions, J. Geophys. Res.-Space, 119, 8499-8511, doi:10.1002/2013JA019457, 2014.

Schlatter, N. M., Belyey, V., Gustavsson, B., Ivchenko, N., Whiter, D., Dahlgren, H., Tuttle, S., and Grydeland, T.: Auroral ion acoustic wave enhancement observed with a radar interferometer system, Ann. Geophys., 33, 837-844, doi:10.5194/angeo-33837-2015, 2015.

Shepherd, M. G., McConnell, J. C., Tobiska, W. K., Gladstone, G. R., Chakrabarti, S., and Schmidtke, G.: Inference of atomic oxygen concentration from remote sensing of optical aurora, J. Geophys. Res., 100, 17415-17428, doi:10.1029/94JA03374, 1995.

Sullivan, J. M., Lockwood, M., Lanchester, B. S., Kontar, E. P., Ivchenko, N., Dahlgren, H., and Whiter, D. K.: An optical study of multiple NEIAL events driven by low energy electron precipitation, Ann. Geophys., 26, 2435-2447, doi:10.5194/angeo-262435-2008, 2008. 